\title{
Experiments and Modeling of Si-Ge Interdiffusion with Partial Strain Relaxation in Epitaxial SiGe Heterostructures
}

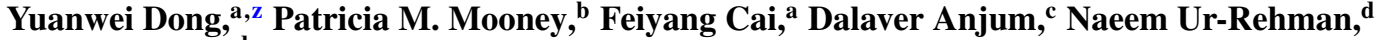 \\ Xixiang Zhang, ${ }^{\mathrm{d}}$ and Guangrui (Maggie) Xia ${ }^{\mathrm{a}}$
}

\author{
${ }^{a}$ Department of Materials Engineering, University of British Columbia, Vancouver, BC V6T1Z4, Canada \\ ${ }^{b}$ Physics Department, Simon Fraser University, Burnaby, BC V5A 1S6, Canada \\ ${ }^{c}$ Advanced Imaging and Characterization Lab, King Abdullah University of Science \& Technology (KAUST), \\ Thuwal, Makkah 23599, Saudi Arabia \\ ${ }^{d}$ Division of Physical Sciences and Engineering, King Abdullah University of Science \& Technology (KAUST), \\ Thuwal 23955-6900, Saudi Arabia
}

\begin{abstract}
$\mathrm{Si}-\mathrm{Ge}$ interdiffusion and strain relaxation were studied in a metastable SiGe epitaxial structure. With Ge concentration profiling and ex-situ strain analysis, it was shown that during thermal anneals, both Si-Ge interdiffusion and strain relaxation occurred. Furthermore, the time evolutions of both strain relaxation and interdiffusion were characterized. It showed that during the ramp-up stage of thermal anneals at higher temperatures $\left(800^{\circ} \mathrm{C}\right.$ and $\left.840^{\circ} \mathrm{C}\right)$, the degree of relaxation, $R$, reached a "plateau", while interdiffusion was negligible. With the approximation that the $R$ value is constant after the ramp-up stage, a quantitative interdiffusivity model was built to account for both the effect of strain relaxation and the impact of the relaxation induced dislocations, which gave good agreement with the experiment data.

(C) 2014 The Electrochemical Society. [DOI: 10.1149/2.0041410jss] All rights reserved.
\end{abstract}

Manuscript submitted May 28, 2014; revised manuscript received July 9, 2014. Published July 26, 2014.

As electronic and optoelectronic devices are continuously scaled down for better performance, novel materials and process techniques have been developed and integrated into the state-of-the-art semiconductor device manufacturing. In the last decades, $\operatorname{Si}_{l-x} \mathrm{Ge}_{x}(0 \leq x \leq 1)$ alloys have become key materials in electronic devices. Examples are channel materials in p-type metal-oxide-semiconductor field-effect transistors (MOSFETs) for higher hole mobilities, ${ }^{1,2}$ the tunneling layer in tunneling FETs, ${ }^{3}$ and the quantum wells in resonant tunneling diodes. ${ }^{4}$ For optoelectronic applications, SiGe alloys and Ge are also employed in modulators, ${ }^{5} \mathrm{Ge}$ photodiodes ${ }^{6,7}$ and $\mathrm{Ge} / \mathrm{Si}$ lasers. ${ }^{8,9}$ $\mathrm{Si} / \mathrm{Si}_{l-x} \mathrm{Ge}_{x}$ and $\mathrm{Si}_{1-x} \mathrm{Ge}_{x} / \mathrm{Si}_{1-y} \mathrm{Ge}_{y}$ heterostructures with abrupt changes in Ge concentration have become key structures for many electronic and optoelectronic devices.

During the fabrication of those devices, especially the ones requiring high temperature processes, unavoidably, Si and Ge atoms interdiffuse at the interface between two layers in heterostructures. This interdiffusion increases exponentially with Ge concentration and compressive stress, and also increases with ion implantation damage. ${ }^{10,11}$ Si-Ge interdiffusion is generally undesirable as it degrades MOSFET performance by reducing strain and carrier confinement and increasing alloy scattering. ${ }^{12}$ It also decreases photodetector efficiency, ${ }^{13}$ and delays the lasing of Ge/Si lasers. ${ }^{14}$ Therefore, understanding Si-Ge interdiffusion behavior under different strain conditions is a topic with great technological significance. In Dong et al's previous studies, a unified Si-Ge interdiffusivity model was built for Si-Ge interdiffusion without strain over the full Ge fraction range and was validated with experiments. ${ }^{15}$ In addition, the role of compressive strain in $\mathrm{Si}-\mathrm{Ge}$ interdiffusion was modeled based on experimental data in Dong et al's recent work. ${ }^{16}$ However, until now, there have been few studies available on Si-Ge interdiffusion behavior with partial compressive strain relaxation.

This issue is of great relevance to the semiconductor industry, as strain relaxation is very common in semiconductor devices due to thin film thicknesses, ${ }^{17,18}$ high Ge fractions, ${ }^{19}$ high temperature anneals and ion implantation damage ${ }^{20,21}$ etc. Thus, in this work $\mathrm{Si}-\mathrm{Ge}$ interdiffusion under partial strain relaxation was investigated. Strain relaxation was characterized using high resolution X-ray diffraction (HRXRD) and a quantitative model was built for $\mathrm{Si}-\mathrm{Ge}$ interdiffusion with partial strain relaxation over a wider Ge fraction range. This model was shown to produce accurate predictions of Ge peak positions in comparison with experimental data.

\section{Experimental}

Epitaxial structure growth and annealing.- A multilayered epitaxial structure $\mathrm{Si}_{0.55} \mathrm{Ge}_{0.45} / \mathrm{Si}_{0.17} \mathrm{Ge}_{0.83} / \mathrm{Si}_{0.55} \mathrm{Ge}_{0.45}$ was used for this study, as shown in Figure 1. The $\mathrm{Si}_{0.17} \mathrm{Ge}_{0.83}$ layer is under the biaxial compressive strain. This structure is labeled as S4583. The epitaxial layers were grown on a 6 inch (100) Czochralski (CZ) p-type Si wafer in an Applied Materials "Epi Centura" system. In order to minimize the threading dislocation density (TDD) and its impact on Si-Ge interdiffusion in the top layers, a $5 \mu \mathrm{m}$ graded relaxed SiGe buffer (GRB) layer was grown on the $\mathrm{Si}$ substrate. The Ge fraction is graded linearly from 0 to 0.45 . Next a $1 \mu \mathrm{m}$ strain-relaxed $\mathrm{Si}_{0.55} \mathrm{Ge}_{0.45}$ layer was grown on the GRB layer at $900^{\circ} \mathrm{C}$ as the virtual substrate, to make sure the $\mathrm{Si}_{0.17} \mathrm{Ge}_{0.83}$ layer of interest is far enough from the defected GRB layer. The TDD in the as-grown relaxed $\mathrm{Si}_{0.55} \mathrm{Ge}_{0.45}$ layer was measured using the etch pit density (EPD) technique, and was estimated to be on the order of $10^{4} \mathrm{~cm}^{-2}$. Next, a thin compressively strained $\mathrm{Si}_{0.17} \mathrm{Ge}_{0.83}$ layer was grown at $365^{\circ} \mathrm{C}$. Its thickness $(9 \mathrm{~nm})$ was designed to be larger than the Matthews-Blakeslee equilibrium critical thickness $(5 \mathrm{~nm})$, resulting in a metastable layer. It tends to relax during thermal anneals. ${ }^{18}$ On top of this compressively strained layer, another relaxed $\mathrm{Si}_{0.55} \mathrm{Ge}_{0.45}$ was grown at $525^{\circ} \mathrm{C}$. Finally, a thin silicon cap was grown on top at $600^{\circ} \mathrm{C}$. The nominal thicknesses of the top strained $\mathrm{Si}$, relaxed $\mathrm{Si}_{0.55} \mathrm{Ge}_{0.45}$ and compressive $\mathrm{Si}_{0.17} \mathrm{Ge}_{0.83}$ layers are $5 \mathrm{~nm}, 30 \mathrm{~nm}$ and $9 \mathrm{~nm}$ respectively. The sample structure is shown in Figure 1.

Inert anneals were performed in nitrogen ambient using an enclosed Linkam TS1200 high temperature heating stage. The annealing condition design criteria were: 1) to obtain enough diffusion for SIMS measurements and introduce sufficient strain relaxation for HRXRD to detect, and 2) diffusion should be limited such that surface transport is insignificant and can be ignored, so the mass transport is only by interdiffusion governed by Fick's laws. A few diffusion tests were performed. The anneals at $760^{\circ} \mathrm{C}, 800^{\circ} \mathrm{C}$ and $840^{\circ} \mathrm{C}$ for 40 mins satisfied the above criteria. To investigate the time dependence of the strain relaxation and $\mathrm{Si}-\mathrm{Ge}$ interdiffusion, four anneals for different times were performed at each temperature. The annealing conditions and the sample naming convention are the following: as-grown (AG), ramp up and down only (RUDO), 10-minute anneal with ramp up and down (RUD10), 20-minute anneal with ramp up and down (RUD20) and 40-minute anneal with ramp up and down (RUD40). The ramp-up and ramp down rates are $40^{\circ} \mathrm{C} / \mathrm{min}$ and $100^{\circ} \mathrm{C} / \mathrm{min}$ respectively. The temperature profiles at $800^{\circ} \mathrm{C}$ are shown in Figure 2 .

Depth profiling of Ge and strain analysis.- Secondary ion mass spectrometry (SIMS) measurements were performed by Evans 


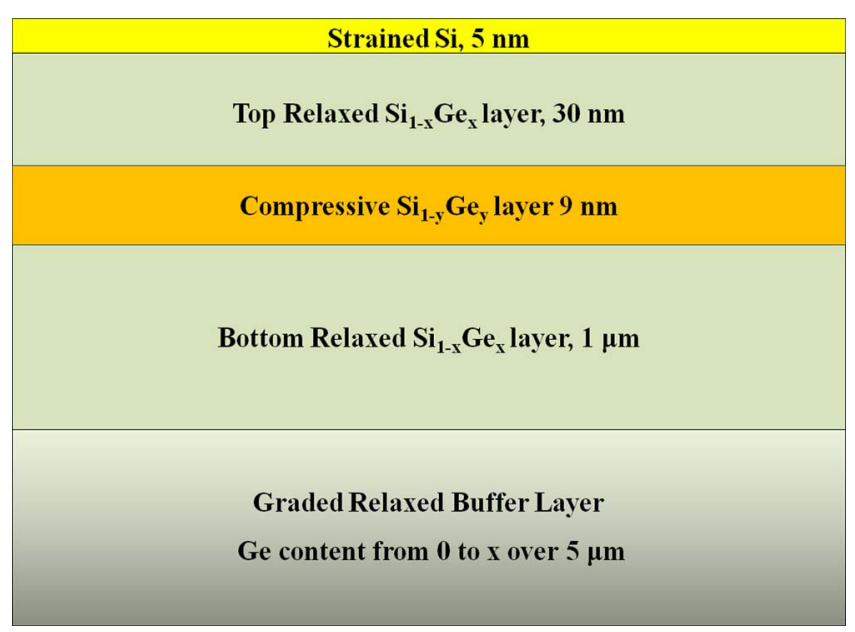

Figure 1. Schematic structure of S4583.

Analytical Group to obtain the Ge profiles in the as-grown and annealed samples. The samples were sputtered with $1 \mathrm{keV} \mathrm{Cs}^{+}$primary ion beam obliquely incident on the samples at $60^{\circ}$ off the sample surface normal. The sputter rate was calibrated using stylus profilometer measurements of total sputtered crater depths, and corrected on a point-by-point basis for the known sputter rate variation with $\mathrm{SiGe}$ composition. The measurement uncertainty in Ge fraction is \pm 1 at. $\%$. The depth uncertainty for a profiling depth of $100 \mathrm{~nm}$ is $2 \mathrm{~nm}$.

High resolution X-ray diffraction (HRXRD) measurements were performed to characterize the biaxial compressive strain and the degree of relaxation $(R)$ in the compressive $S i_{1-y} G e_{y}$ and bottom $S i_{1-x} G e_{x}$ layers of the as-grown and annealed samples. All the measurements were performed using a PANalytical X'Pert PRO MRD with a triple axis configuration. Symmetric (004 Bragg reflection) and asymmetric (115 Bragg reflection) scans were performed to obtain both the in-plane and out-of-plane lattice constants of the compressive $S i_{1-y} G e_{y}$ layer and the bottom relaxed $S i_{1-x} G e_{x}$ layer. This allows us to determine both the Ge fraction and strain in these layers. To eliminate the influence of the substrate wafer miscut and the tilt of the epitaxial films, XRD scans were performed with the wafer

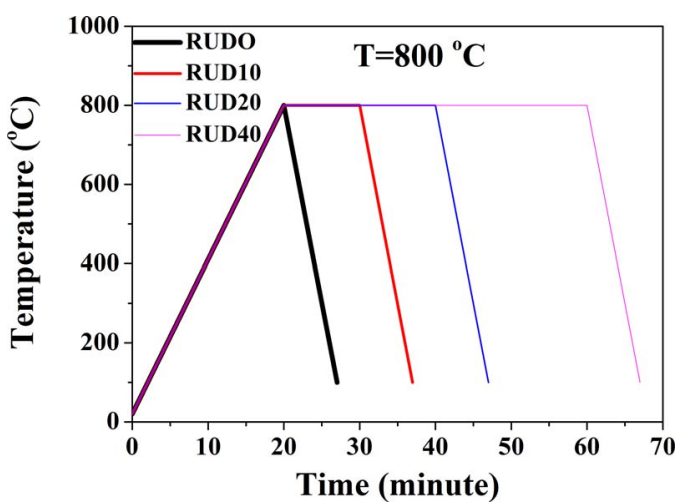

Figure 2. Temperature profiles of the four anneal scenarios at $800^{\circ} \mathrm{C}$ labeled as RUDO, RUD10, RUD20 and RUD40.

oriented at phi $=0^{\circ}$ and $180^{\circ}$ for both symmetric and asymmetric reflections, ${ }^{22,23}$ where "phi" denotes the angle rotation of the sample about its surface normal. The Ge fraction and strain of the $S i_{1-y} G e_{y}$ and bottom relaxed $S i_{1-x} G e_{x}$ layers were extracted by matching the average peak separation between the peaks of the layer and substrate with that simulated by the PANalytical Epitaxy software package. ${ }^{24}$ The thicknesses of the $S i_{1-y} G e_{y}$ layers used for those simulations are the full width at half maximum (FWHM) of the corresponding SIMS profiles.

\section{Results and Discussion}

Ge depth profiles measured by SIMS. - The as-grown and the annealed Ge profiles of the samples annealed at three different temperatures are shown Figure 3a. The anneal time is 40 mins for all temperatures. The Ge peak of the as-grown $S i_{1-y} G e_{y}$ layer has a triangle-like shape, since interdiffusion occurred during the growth of the top two layers after the $S i_{1-y} G e_{y}$ layer was grown. In addition, there was partial strain relaxation at the same time, as shown in Table I. The as-grown maximum Ge fraction is 0.83 . This interdiffusion during the growth is hard to minimize due to the large $\mathrm{Ge}$ fraction and high compressive strain in the $S i_{1-y} G e_{y}$ layer. Simulations were conducted using two established models in Dong et al's previous
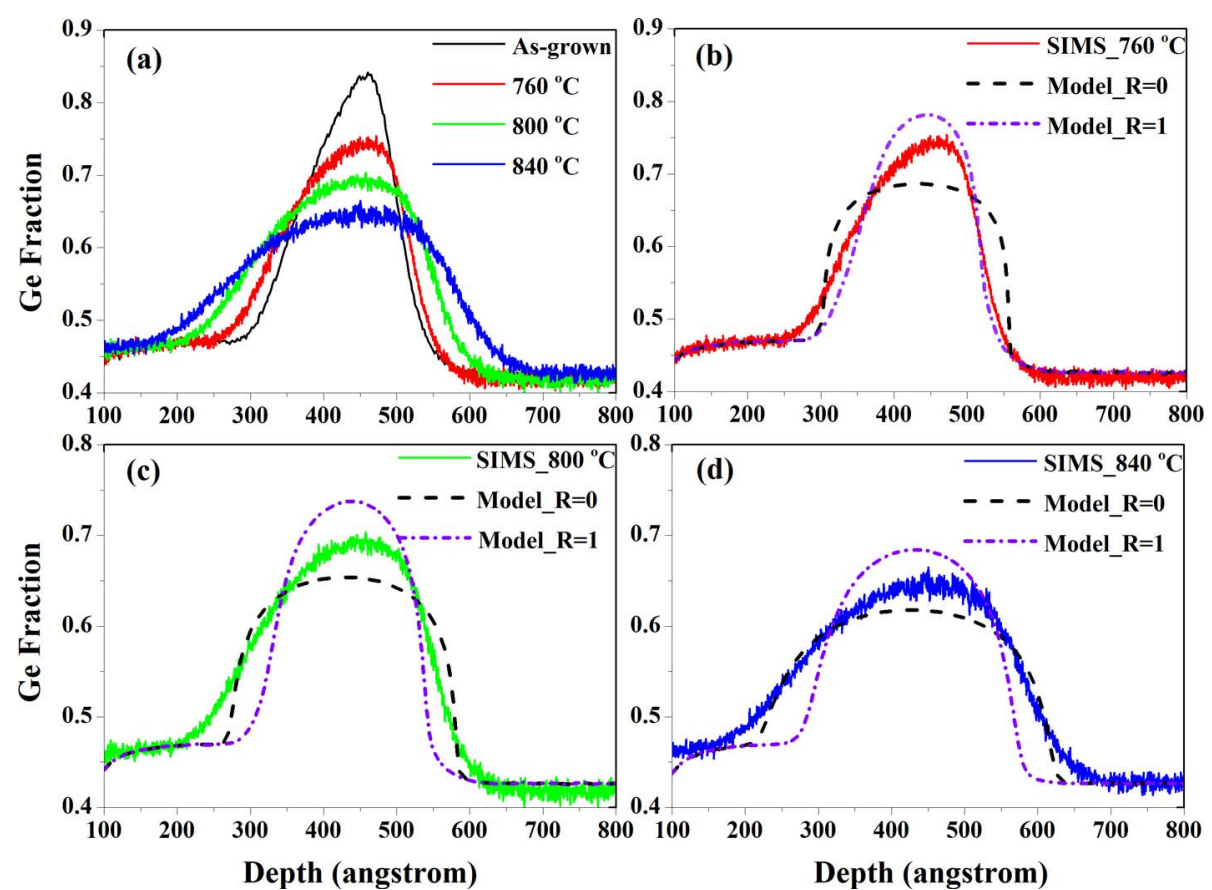

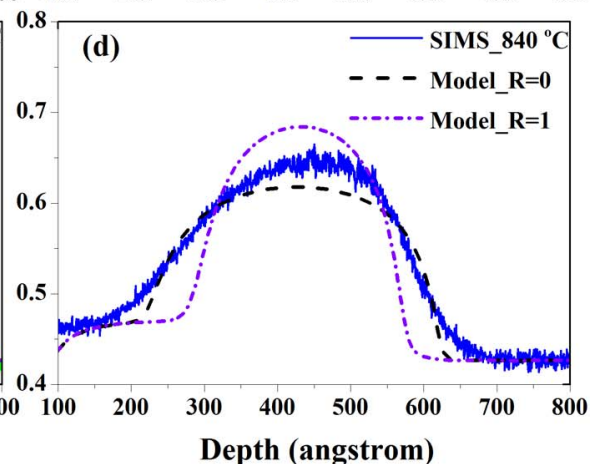

Figure 3. (a) Ge profiles measured by SIMS for as-grown and annealed samples. The anneal time is 40 minutes for all temperatures. (b) SIMS profile of $760^{\circ} \mathrm{C}$ annealed sample in comparison with model predictions. (c) SIMS profile of $800^{\circ} \mathrm{C}$ annealed sample in comparison with model predictions. (d) SIMS profile of $840^{\circ} \mathrm{C}$ annealed sample in comparison with model predictions. 
Table $I$. Ge fraction and the degree of relaxation $R$ in the compressive $S i_{1-y} G_{y}$ layers and the bottom $\mathrm{Si}_{1-x} \mathrm{Ge}_{x}$ layers from XRD measurements. The error bars of the Ge fraction and the $R$ value are \pm 0.01 and \pm 5 respectively for $S i_{1-y} G e_{y}$ layers, and \pm 0.005 and \pm 0.5 respectively for the bottom $\mathrm{Si}_{1-x} \mathbf{G e}_{x}$ layers.

\begin{tabular}{|c|c|c|c|c|}
\hline \multirow[b]{2}{*}{ Thermal condition } & \multicolumn{2}{|c|}{$\mathrm{Si}_{1-y} \mathrm{Ge}_{y}$ layer } & \multicolumn{2}{|c|}{$\begin{array}{l}\text { Bottom relaxed } \\
S i_{1-x} G e_{x} \text { layer }\end{array}$} \\
\hline & $y$ & $\mathrm{R}(\%)$ & $x$ & $\mathrm{R}(\%)$ \\
\hline As-grown & 0.840 & 10 & 0.422 & 97.0 \\
\hline $760^{\circ} \mathrm{C}$ for 40 minutes & 0.734 & 45 & 0.418 & 96.5 \\
\hline $800^{\circ} \mathrm{C}$ for 40 minutes & 0.683 & 45 & 0.425 & 96.0 \\
\hline $840^{\circ} \mathrm{C}$ for 40 minutes & 0.622 & 50 & 0.430 & 96.0 \\
\hline
\end{tabular}

work, shown in Figure 3b 3d. One model is the unified model for fully relaxed $\mathrm{SiGe}$ (equivalently $R=1$ ) built over the full Ge fraction range in Ref. 15, and the other is the interdiffusivity model with full compressive strain (equivalently $R=0$ ) in Ref. 16. The SIMS profiles fall in between the model predictions under these two extreme strain conditions, which indicates that the strain has partially relaxed during the thermal anneals.

At each temperature, the samples were also annealed for different times: RUDO, RUD10, RUD20 and RUD40, as illustrated in Figure 2. The Ge profiles of the samples annealed with different times at $800^{\circ} \mathrm{C}$ are shown in Figure 4. Comparing the SIMS profile of the RUDO sample with the as-grown one, we can see that $\mathrm{Si}-\mathrm{Ge}$ interdiffusion is negligible during the ramp up and down stages, where the peak decreased by about 1 at.\%, which is within the SIMS measurement uncertainty of $\mathrm{Ge}$ fractions. If we compare the Ge peaks after the 10 , 20 and 40-minute diffusion, we see that the Ge peak drop in the first 10 minutes is much larger than that in the next 10 minutes and the next 30 minutes. As the annealing time increased, the rate of the peak drop in the $\mathrm{Si}_{1-y} \mathrm{Ge}_{y}$ layer became slower, which is attributed to the strong concentration and strain dependence of Si-Ge interdiffusivity. The interdiffusivity became much smaller as the Ge fraction decreased during annealing. Accordingly, the rate of the Ge peak drop was slower as the diffusion time increased.

Ge fraction and strain analysis by HRXRD.- In Figure 5, the 004 symmetric and 115 asymmetric XRD scans from samples annealed at the three different temperatures for 40 minutes are shown. The strongest peak is from the $\mathrm{Si}$ substrate. The second strongest peak is from the thick bottom $S i_{1-x} G e_{x}$ layer. The peaks from the compressive $S i_{1-y} G e_{y}$ layers are relatively broad due to their shape and small thickness. There are no noticeable peaks from the top $S i_{1-x} G e_{x}$ layer and the Si cap layer, due to their small thicknesses. Using the Si substrate peak as a reference, we can see that the peaks from the $S i_{1-y} G e_{y}$ layers shift to the right and become more in-

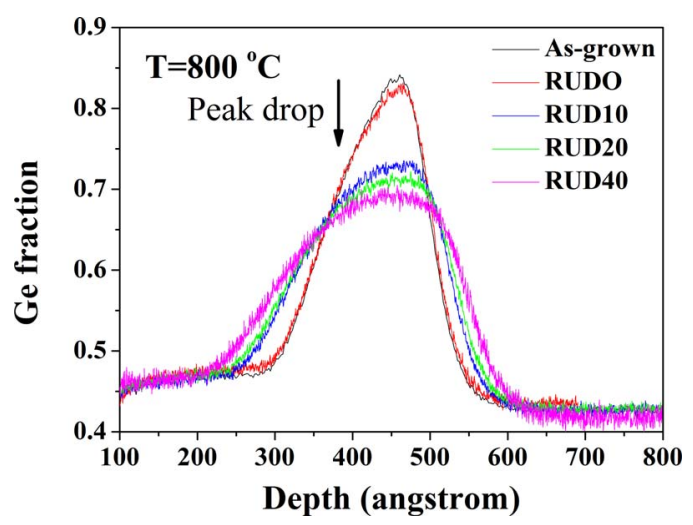

Figure 4. Ge profiles measured by SIMS for as-grown and annealed samples with different anneal times at $800^{\circ} \mathrm{C}$.
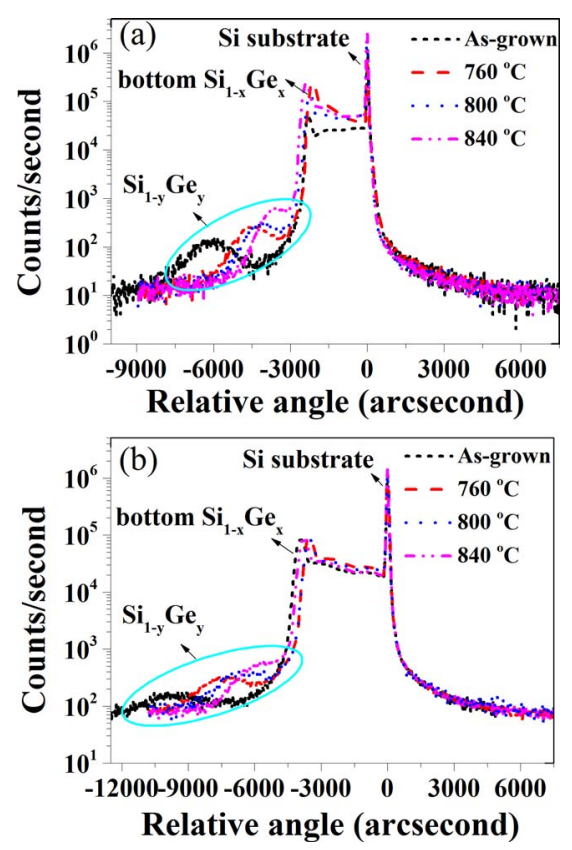

Figure 5. (a) 004 symmetric XRD and (b) 115 asymmetric scans at phi $=0^{\circ}$ from S4583 samples. The most intense narrow peak is from the Si substrate. The second strongest peak is from the bottom $S i_{1-x} G e_{x}$ layer. The peak on the far left is from the thin $S i_{1-y} G e_{y}$ layer.

tense and narrower as temperature increased. This indicates that the $S i_{1-y} G e_{y}$ layer became thicker and had a lower Ge fraction and lower strain after anneals, as expected due to $\mathrm{Si}-\mathrm{Ge}$ interdiffusion and strain relaxation.

The degree of relaxation $R$ after annealing depends on multiple factors such as the Ge fraction, layer thickness, as-grown relaxation, and the anneal temperature and time..$^{25-27}$ Based on the peak positions from the symmetric and asymmetric scans at phi $=0^{\circ}$ and $180^{\circ}$, the Ge fraction and strain in the $\mathrm{Si}_{1-y} \mathrm{Ge}_{y}$ and bottom $\mathrm{Si}_{1-x} \mathrm{Ge}_{x}$ layers were extracted by simulations with the PANalytical Epitaxy software package, ${ }^{24}$ which are summarized in Table I. The $R$ values of the bottom $\mathrm{Si}_{1-x} \mathrm{Ge}_{x}$ layers are around $96 \%$, which indicates that the bottom $\mathrm{Si}_{1-x} \mathrm{Ge}_{x}$ layers are very close to full relaxation. There is about $10 \%$ relaxation in the as-grown metastable $\mathrm{Si}_{1-y} \mathrm{Ge}_{y}$ layer, and after annealing the $R$ value of this layer increased up to 50\% relaxation, depending on the thermal budget. Consistent with the SIMS profiles, the Ge fraction measured by XRD also decreased, indicating that both interdiffusion and strain relaxation occurred in the metastable $\mathrm{Si}_{1-y} \mathrm{Ge}_{y}$ layer of $\mathrm{S} 4583$ during annealing.

In addition, the time dependence of the $R$ values for all the three annealing temperatures was characterized by the HRXRD and is shown in Figure 6. From Figure 6, we can see that the $R$ value increased greatly at first, and went up to around $45 \%$ and gradually saturated with the annealing time, forming a plateau. The shape of the time dependence of $R$ in S4583 is consistent with the results of the in-situ XRD measurements reported by Fischer and Zaumseil. ${ }^{26}$ At $760^{\circ} \mathrm{C}$, the relaxation rate was slower than the other two temperatures, and the $R$ value reached a plateau at RUD10. In contrast, for the anneals at $800^{\circ} \mathrm{C}$ and $840^{\circ} \mathrm{C}$, the $R$ value almost reached a plateau after the rampup stage. Considering the slower ramp-up rate $\left(40^{\circ} \mathrm{C} / \mathrm{min}\right)$ compared to the ramp-down one $\left(100^{\circ} \mathrm{C} / \mathrm{min}\right)$, it is reasonable to assume that the strain relaxation happened predominantly in the ramp-up stage. After that, the $R$ value only increased by small amounts as the anneal time increases.

Based on the SIMS data and the time dependence of $R$ and SiGe interdiffusion discussed above, we can see that for higher anneal temperatures $\left(800^{\circ} \mathrm{C}\right.$ and $\left.840^{\circ} \mathrm{C}\right)$, the degree of relaxation $R$ reached a plateau after the ramp-up stage, when there was negligible $\mathrm{Si}-\mathrm{Ge}$ 


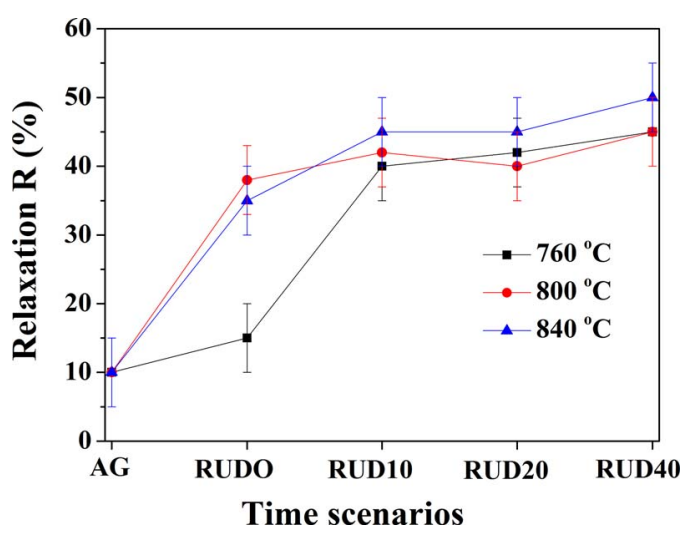

Figure 6. Time dependence of the $R$ values at the three different annealing temperatures. At each temperature, there are five annealing time scenarios: AG, RUDO, RUD10, RUD20 and RUD40.

interdiffusion. In other words, strain relaxation happened before $\mathrm{Si}-\mathrm{Ge}$ interdiffusion in samples annealed at $800^{\circ} \mathrm{C}$ and $840^{\circ} \mathrm{C}$.

\section{Interdiffusivity Modeling and Discussion}

Modeling of lattice-mediated Si-Ge interdiffusion with partial strain relaxation. - In Dong et al's recent work, ${ }^{16}$ we studied similar epitaxial structures with high compressive strain, which was reduced only by $\mathrm{Si}-\mathrm{Ge}$ interdiffusion, with structure coherency maintained. To avoid confusion, in this work, strain relaxation only refers to plastic relaxation via dislocations, not to strain reduction by $\mathrm{Si}-\mathrm{Ge}$ interdiffusion. The degree of relaxation, $R$, by the introduction of misfit dislocations at the interfaces of heterostructures ${ }^{28}$ can be expressed as

$$
R=\frac{a-a_{s}}{a_{r}-a_{s}} \times 100 \%,
$$

where $a, a_{r}$ and $a_{s}$ are the in-plane lattice parameters of an epitaxial layer with partial strain relaxation, the epitaxial layer material with full strain relaxation and the substrate respectively.

For biaxially strained layers with partial strain relaxation grown on virtual substrates,${ }^{26}$ the biaxial strain $\epsilon$ of a partially relaxed layer is given by

$$
\varepsilon=\varepsilon_{0}(1-R)
$$

where $\varepsilon_{0}$ is the lattice mismatch strain in a coherently strained epitaxial film. For an epitaxial $\mathrm{Si}_{1-y} \mathrm{Ge}_{y}$ film on $\mathrm{aSi}_{1-x} \mathrm{Ge}_{x}$ virtual substrate, the biaxial lattice mismatch strain can be empirically expressed as $\varepsilon_{0}=-0.418(y-x)$.

Compressive strains play two main roles in Si-Ge interdiffusion: ${ }^{16}$ thermodynamically, strain energy contributes to the driving force, and strain can change interdiffusivity itself. For strained multilayered $\mathrm{SiGe}$ structures, the strain energy contributes to the total free energy. Then the total Gibbs free energy per mole at the Ge fraction $x_{G e}$ includes two dominant terms, shown in Equation 3.

$$
G_{\text {total }}=G_{c}+G_{e},
$$

where $G_{\text {total }}$ is the total Gibbs free energy per mole. $G_{c}$, and $G_{e}$ denote the free energy contributions from chemical mixing and elastic biaxial strain.

$\mathrm{Si}$ and Ge both belong to Group IVA in the periodic table. They have same outmost electron distribution, so their chemical properties are similar, and they can form stable random alloy solutions over the full Ge fraction range, which obeys regular solution theory very closely at high temperatures $(>200 \mathrm{~K}) .^{29,30}$ Thus, following the regular solution treatment by Cahn and Hilliard, ${ }^{31,32}$ those two free energy terms can be expressed as Equation 4 and 5. The details of the deriva- tion of these equations were well conducted in Ref. 31 and 32.

$$
\begin{aligned}
G_{c}= & G_{0}+\alpha x_{G e}\left(1-x_{G e}\right)+R T\left[x_{G e} \ln x_{G e}\right. \\
& \left.+\left(1-x_{G e}\right) \ln \left(1-x_{G e}\right)\right],
\end{aligned}
$$

where $G_{0}, \alpha, R, T$ and $x_{G e}$ are the reference free energy, the interaction factor for $\mathrm{SiGe}$ system, the ideal gas constant, absolute temperature and $\mathrm{Ge}$ fraction. For a biaxially strained thin $\mathrm{Si}_{1-\mathrm{x}} \mathrm{Ge}_{\mathrm{x}}$ film,

$$
G_{e}=\frac{1}{2} V_{m} \sum_{i}^{2} \varepsilon_{i} \sigma_{i},
$$

where $\varepsilon_{i}$ and $\sigma_{i}$ are the biaxial strain and stress; $V_{m}$ is the molar volume of the solid solution, for SiGe, $V_{m} \approx 13 \mathrm{~cm}^{3} / \mathrm{mol}$. Combining Equation 2 and 5, the strain energy can be ideally expressed as

$$
G_{e}=\frac{1}{2} V_{m} \sum_{i}^{2} \varepsilon_{i} \sigma_{i}=V_{m} Y_{U V W}(1-R)^{2} \varepsilon_{0}^{2},
$$

where $\sigma_{i}=Y_{U V W} \varepsilon_{i}$, and $Y_{U V W}$ is the biaxial modulus, which equals $E /(1-v) . E$ is Young's modulus, and $v$ is Poisson ratio. $Y_{U V W}$ is calculated with the method used by Aziz et al. ${ }^{33}$ For our samples, $\langle U V W\rangle$ is $\langle 100\rangle$.

In order to obtain the equation of interdiffusion flux, Greer and Spaepen introduced a concept, $\Delta G_{\text {total }}=\frac{\partial G_{\text {total }}}{\partial x_{G e}}$, which is the change in free energy on reversibly replacing $\mathrm{Ge}$ and $\mathrm{Si}$ atoms. ${ }^{34}$ Thus, the interdiffusion flux along the $z$ direction normal to the sample surface can be expressed as

$$
\tilde{J}=-C_{\text {total }} M \frac{\partial \Delta G_{\text {total }}}{\partial z}=-M \frac{\partial \Delta G_{\text {total }}}{\partial x_{G e}} \frac{\partial C_{G e}}{\partial z}=-M g^{\prime \prime} \frac{\partial C_{G e}}{\partial z},
$$

where $C_{\text {total }}, \mathrm{M}$ and $C_{G e}$ denote the total concentration of $\mathrm{Si}$ and $\mathrm{Ge}$ atoms, mass mobility and Ge concentration, and $g^{\prime \prime}=\frac{\partial \Delta G_{\text {total }}}{\partial x_{G e}}=$ $\frac{\partial^{2} G_{\text {total }}}{\partial x_{G e}^{2}}$, the second derivative of the total free energy over $x_{G e}$.

Combining the Equation 3-7, the interdiffusion flux with a constant $R$ can be expressed as

$$
\begin{aligned}
\tilde{J} & =-M G_{c}^{\prime \prime}\left[1+\frac{2 V_{m} \eta^{2}(1-R)^{2} Y_{U V W}}{G_{c}^{\prime \prime}}\right] \frac{\partial C_{G e}}{\partial z} \\
& =-\tilde{D}^{\text {strained }}\left[1+\frac{2 V_{m} \eta^{2}(1-R)^{2} Y_{U V W}}{G_{c}^{\prime \prime}}\right] \frac{\partial C_{G e}}{\partial z}
\end{aligned}
$$

where $G_{c}^{\prime \prime}$ is the second derivative of the Gibbs free energy from chemical mixing over $x_{G e} ; \eta$ is the linear expansion coefficient (for $\mathrm{Si}$ and Ge, $\eta$ is 0.0418 ); $\tilde{D}^{\text {strained }}=M G_{c}^{\prime \prime}$, which is the interdiffusivity under biaxial strain and reflects the impact of the biaxial strain only on the interdiffusivity itself without the driving force impact.

In the right parentheses of Equation 8, "1" reflects the contribution from chemical mixing, and the second term $\frac{2 V_{m} \eta^{2}(1-R)^{2} Y_{U V W}}{G_{c}^{\prime \prime}}$ reflects the magnitude of the biaxial strain energy contribution to the driving force, which depends on temperature and degree of relaxation $R$. In addition, $\tilde{D}^{\text {strained }}$ has been well modeled in our recent work, ${ }^{16}$ and can be expressed as

$$
\tilde{D}^{\text {strained }}=\tilde{D}^{\text {relax }} e^{\frac{-q^{\prime} \varepsilon}{k T}}
$$

where $\tilde{D}^{\text {relax }}$ is the interdiffusivity without strain in Ref. $15, q^{\prime}$ is the strain derivative of interdiffusivity in Ref. 16, and $q^{\prime}=110-$ $0.081 T . T$ is the absolute temperature in Kelvin.

For ease of modeling, comparing the equation 8 to the classic expression of Fick's first law, we can define an apparent diffusivity to include the biaxial strain energy contribution to the driving force. Combining Equation 2, 8 and 9, the apparent interdiffusivity with strain relaxation can be expressed as:

$$
\tilde{D}_{\text {apparent }}=\left[1+\frac{2 V_{m} \eta^{2}(1-R)^{2} Y_{U V W}}{G_{c}^{\prime \prime}}\right] \tilde{D}^{\text {relax }} e^{\frac{-q^{\prime} \varepsilon_{0}(1-R)}{k T}} \equiv \tilde{D}_{\text {lattice }}
$$



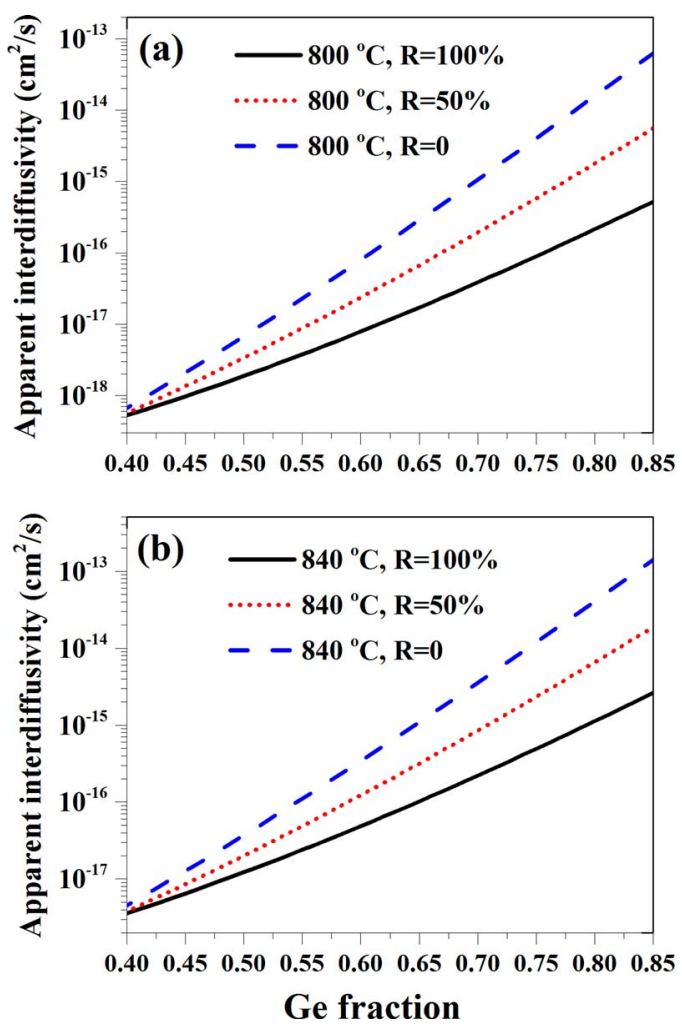

Figure 7. Comparison of $\tilde{D}_{\text {lattice }}$ vs. $x_{G e}$ for $\mathrm{SiGe}$ on a $S i_{0.60} G e_{0.40}$ virtual substrate with different $R$ : (a) at $800^{\circ} \mathrm{C}$ and (b) at $840^{\circ} \mathrm{C}$.

In Equation 10, the degree of relaxation $R$ appears in two places, $(1-R)^{2}$ in the large parenthesis for the driving force change and $(1-R)$ in the exponent term for the interdiffusivity change. When the $R$ value equals 1 , we can see that the apparent interdiffusivity equals $\tilde{D}^{\text {relax }}$, where there is no strain impact. When the $R$ value equals to zero, the apparent interdiffusivity is same as the model with full compressive strain built in Ref. 16. As $\tilde{D}_{\text {apparent }}$ discussed so far is limited to point-defect mediated diffusion, also known as lattice diffusion, we can define $\tilde{D}_{\text {lattice }} \equiv \tilde{D}_{\text {apparent }}$.
The apparent interdiffusivities with different $R$ values at $800^{\circ} \mathrm{C}$ and $840^{\circ} \mathrm{C}$ are compared in Figure 7 . The apparent interdiffusivity when $R=0.5$ is in between those when $R=0$ and $R=1$. As the biaxial compressive strain reduces to $\varepsilon_{0}(1-R)$, correspondingly, the strain enhancement of interdiffusion decreases, which is consistent with the SIMS results in Figure 3. According to the model for interdiffusion with partially relaxed compressive strain in Equation 10, at $800^{\circ} \mathrm{C}$ the interdiffusivity of a $10 \%$ relaxed $\mathrm{Si}_{0.17} \mathrm{Ge}_{0.83}$ (the case of the as-grown sample) is almost 100 times larger than that of a $42 \%$ relaxed $\mathrm{Si}_{0.27} \mathrm{Ge}_{0.73}$ (the case of the RUD10 sample at $800^{\circ} \mathrm{C}$ ). This big difference in interdiffusivity explains why the Ge peak drop is faster during the first 10 minutes in Figure 4.

Model refinement and discussion.- Based on the time dependence of strain relaxation and interdiffusion above, we see that for higher annealing temperatures $\left(800^{\circ} \mathrm{C}\right.$ and $\left.840^{\circ} \mathrm{C}\right)$, the degree of relaxation $R$ reached a plateau after the ramp-up stage. Therefore, for anneals with 10 mins and longer time at the peak temperature, $R$ can reasonably be assumed to be a constant during interdiffusion as the thermal budget in the ramp-up stage is negligible for interdiffusion. Therefore, Si-Ge interdiffusion can be simulated using the model in Equation 10 with a constant $R$, and the model was implemented in TSUPREM- $4,{ }^{35}$ an industry mainstream two-dimension finite element simulation tool. For annealing at $800^{\circ} \mathrm{C}$ with different anneal times (10 minutes, 20 minutes and 40 minutes), an average $R$ value of $42 \%$ is used; while for the anneal at $840^{\circ} \mathrm{C}$ with 40 minutes, an average $R$ value of $47 \%$ is used. For the $760^{\circ} \mathrm{C}$ anneals, however, the $R$ value reached a plateau at RUD10, which means that strain relaxation mostly occur in the first 10 mins anneal after the ramp-up stage, and $R$ cannot be treated as a constant after the ramp-up stage. If $R(t)$ is well characterized with much finer time steps, Equation 10 can still be applied with the measured $R(t)$. With the average $R$ values, predictions using the model in Equation 10 can be made for the anneals at $800^{\circ} \mathrm{C}$ and $840^{\circ} \mathrm{C}$. The comparisons between the model predictions and the corresponding SIMS profiles are shown in Figure 8.

From Figure 8, it is demonstrated that the peak drops predicted by the model in Equation 10 match the ones from the SIMS data for all the four anneal conditions within the SIMS measurement error bar. However, in Figure 8, we can also see the peak shapes of the model predictions do not perfectly match the experimental ones, i.e., the rising and falling edges of the simulated peaks are steeper than those of the SIMS profiles. The steep edges of the model predictions are caused

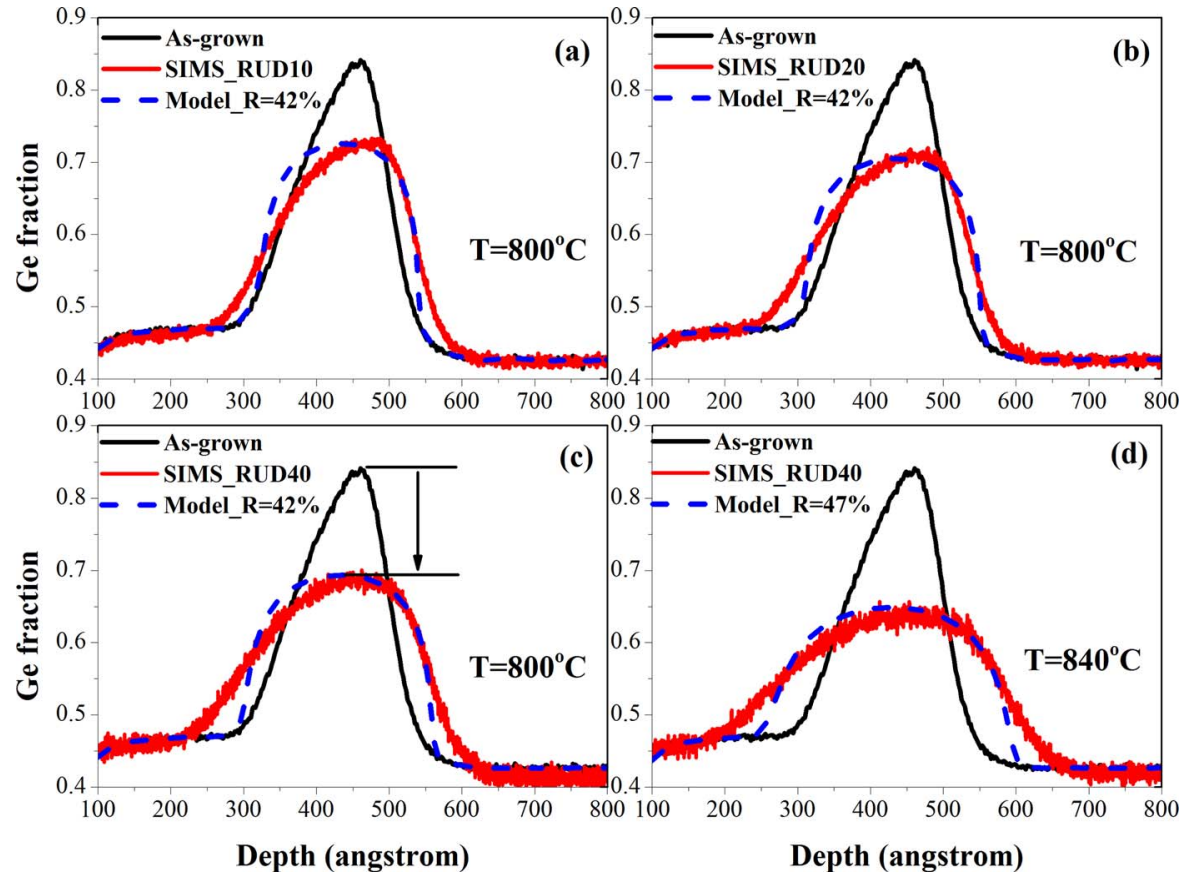

Figure 8. Comparisons between the model predictions and SIMS profiles: (a) for RUD10 at $800^{\circ} \mathrm{C}$, $R=42 \%$; (b) for RUD20 at $800^{\circ} \mathrm{C}, R=42 \%$; (c) for RUD40 at $800^{\circ} \mathrm{C}, R=42 \%$; (d) for RUD40 at $840^{\circ} \mathrm{C}, R=47 \%$. 


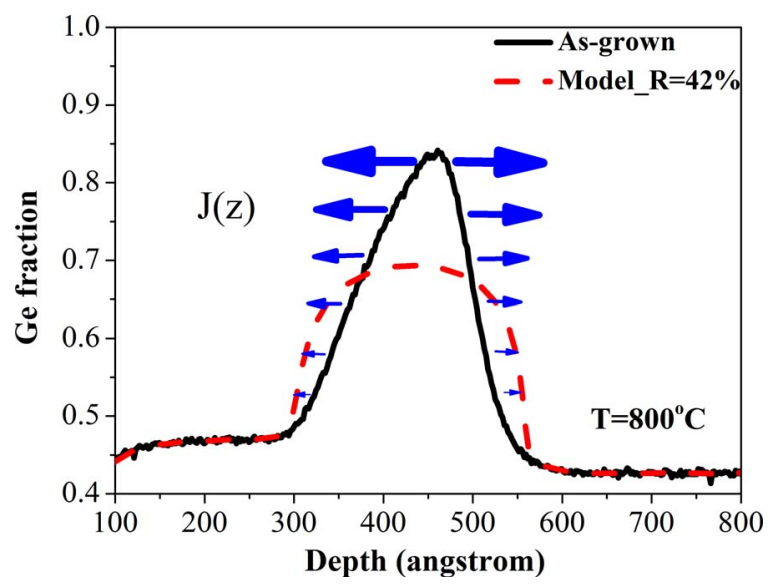

Figure 9. Schematic illustration of the diffusion fluxes at different $\mathrm{Ge}$ concentrations at the starting time point $t=0$, based on the model in Equation 10.

by the strong concentration and compressive stress dependence of $\mathrm{Si}$ Ge interdiffusivity in the model in Equation 10. In 2002, Erdélyi et al demonstrated that if the diffusivity strongly depends on concentration, the interface can become sharper on nanoscale. ${ }^{36}$ Based on Fick's first law, $J=-D \frac{\partial C}{\partial z}$, the diffusion flux is decided by both the diffusivity and the concentration gradient. From the as-grown SIMS profile of S4583, we can see the rising and falling edges are close to linear, so the concentration gradient doesn't change dramatically along the rising and falling edges at time $t=0$. Therefore, the diffusion flux is primarily determined by the interdiffusivity. At the locations with a larger $\mathrm{Ge}$ fraction and thus higher compressive strain and higher interdiffusivity, the diffusion flux is much larger. For S4583, the diffusion flux has a very strong Ge concentration dependence, which is illustrated in Figure 9.

According to the model in Equation 10, at higher Ge concentrations, the diffusion is much faster than at lower Ge concentrations.
As time increases, the rising and falling edges should get steeper. However, this is not what we observed from the SIMS data. The edges of all the SIMS Ge peaks were flatter and went farther than the edges of the simulated Ge peaks, and the Ge peaks did not get steeper as Equation 10 predicted. This issue can be explained by the dislocation-mediated interdiffusion, which is not included in Equation 10. Equation 10 only accounts for the point-defect mediated interdiffusion (lattice diffusion), and the interdiffusivity associated with that is $\tilde{D}_{\text {lattice. }}$. Strain relaxation in SiGe multi-layers is mainly ascribed to the formation of misfit dislocations at the interfaces and their following movements during thermal anneals. ${ }^{23,37,38}$ Misfit dislocations are often associated with threading dislocations, which act as fast diffusion paths. ${ }^{18,39-41}$ The dislocation density is expected to be higher

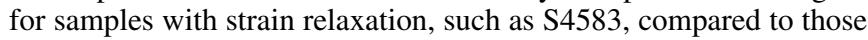
without strain relaxation as in Ref. 16. In order to estimate the collective impact of the relaxation induced dislocations, an additional term $\tilde{D}_{\text {dislocation }}$ is added to $\tilde{D}_{\text {lattice }}$ in calculating the total interdiffusivity $\tilde{D}_{\text {total }}$ as Gavelle et al did for modeling Si-Ge interdiffusion in highly defected Ge/Si heterostructures. ${ }^{42}$ Therefore, the total interdiffusivity can be expressed as

$$
\tilde{D}_{\text {total }}=\tilde{D}_{\text {lattice }}+\tilde{D}_{\text {dislocation }}
$$

where $\tilde{D}_{\text {lattice }}$ is the diffusivity in Equation 10 for lattice mediated interdiffusion, and $\tilde{D}_{\text {dislocation }}$ is the collective diffusivity contributed from relaxation induced dislocations.

In Gavelle et al's study, they used an Arrhenius term to describe the impact of the relaxation induced dislocations on Si-Ge interdiffusion.42 In this work, a similar Arrhenius expression is used to estimate the impact of dislocations analytically.

$$
\tilde{D}_{\text {dislocation }}=D_{0}^{d i s}\left(x_{G e}\right) e^{-\frac{E_{a}^{d i s}}{k T}}
$$

where $D_{0}^{d i s}$ and $E_{a}^{d i s}$ are the prefactor and activation energy respectively. In Ref. $42, D_{0}^{d i s}=0.01 \exp \left(2.5 x_{G e}\right)$ and $E_{a}^{d i s}=3.1 \mathrm{eV}$. "dis" denotes relaxation induced dislocations.

In order to quantify the impact of the dislocations in the tail regions of S4583, the parameters in Equation 12 were extracted by fitting the SIMS profiles, as shown in Figure 10. The extracted prefactor $D_{0}^{\text {dis }}$ is

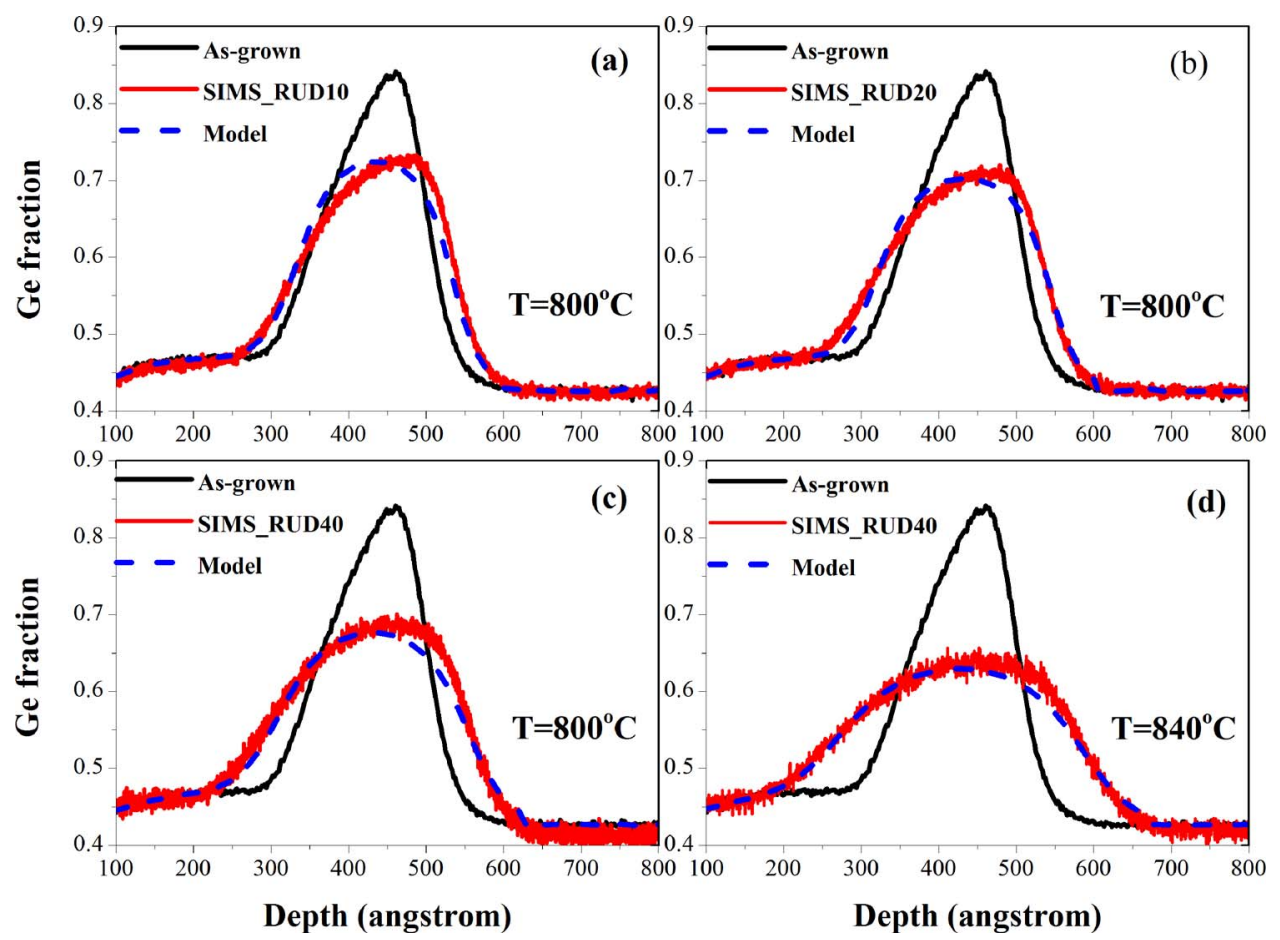

Figure 10. Best fittings to the SIMS profiles for the extraction of $D_{0}^{\text {dis }}$ and $E_{a}^{\text {dis }}$ of $\tilde{D}_{\text {dislocation }}$ : (a) RUD10 at $800^{\circ} \mathrm{C}$; (b) RUD20 at $800^{\circ} \mathrm{C}$; (c) RUD40 at $800^{\circ} \mathrm{C}$ and (d) RUD40 at $840^{\circ} \mathrm{C}$. 


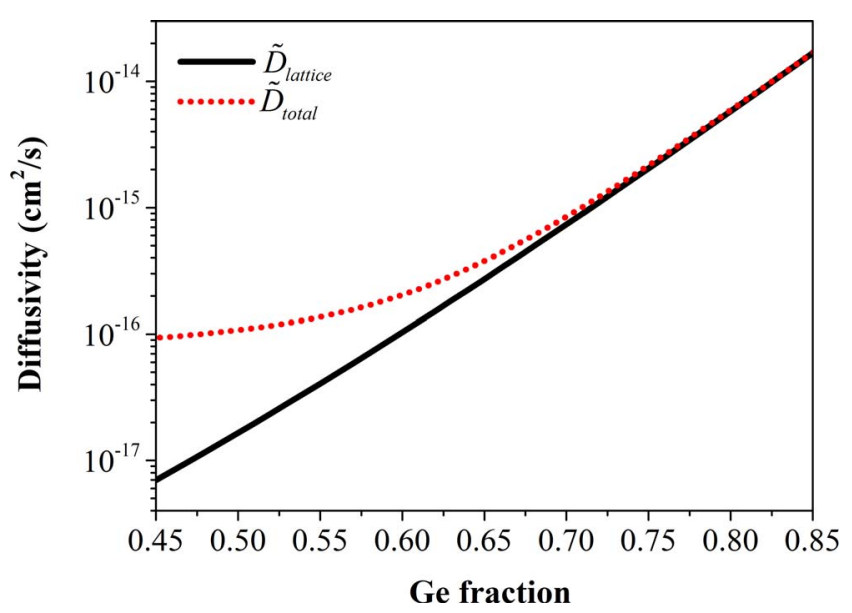

Figure 11. Comparison between the total interdiffusivity $\tilde{D}_{\text {total }}$ and $\tilde{D}_{\text {lattice }}$ for RUD40 at $840^{\circ} \mathrm{C}$.

expressed as $0.017 \exp \left(x_{G e}\right)$ and the activation $E_{a}^{d i s}$ is $3.15 \mathrm{eV}$, and this set of parameters can generate good fittings to all measured SIMS profiles as shown in Figure 10. Compare to the parameters extracted in Ref. 42, where $D_{0}^{d i s}=0.01 \exp \left(2.5 x_{G e}\right)$ and $E_{a}^{d i s}=3.1 \mathrm{eV}$, we can see that the activation energy is very close, but $D_{0}^{\text {dis }}$ is quite different. It should be noted that the structures in Gavelle et al's work in Ref. 42 were highly defected relaxed Ge films on Si. The dislocation density in their samples is much higher than that in this work.

The comparison between the total interdiffusivity $\tilde{D}_{\text {total }}$ and the point-defect mediated interdiffusivity $\tilde{D}_{\text {lattice }}$ is shown in Figure 11. In the relatively lower Ge fraction region, the interdiffusion is strongly affected by the presence of strain-relaxation induced dislocations, and the interdiffusivity can be enhanced by one order of magnitude. However, in the higher Ge fraction region, the total interdiffusivity is dominated by the $\tilde{D}_{\text {lattice }}$ term.

The model built in this work is based on a multi-layered $\mathrm{SiGe}$ structure with biaxial compressive strain relaxation, and the annealing conditions were designed for this structure. However, in practice, for different sample structures and annealing conditions, the time evolution of strain relaxation $R(t)$ can vary greatly. Strain relaxation may happen before, with or after Si-Ge interdiffusion. The most complicated case will be the case where interdiffusion and strain relaxation happen simultaneously. Therefore, the time evolution of strain relaxation $R(t)$ should be understood before applying this model. One can imagine that if $R(t)$ is well characterized with a fine time step, then the degree of relaxation $R$ can be approximated to be constant in a small enough time interval. Therefore, interdiffusion can be roughly estimated with the $\tilde{D}_{\text {lattice }}$ term using Equation 10 . For other advanced thermal annealing, such as spike rapid thermal annealing with much faster ramp rates, the characterization of $R(t)$ can be very challenging. Nonetheless, the model in this work provides a validated theoretical frame work for interdiffusion with strain relaxation.

On the dislocation-mediated interdiffusion, as $\tilde{D}_{\text {dislocation }}$ depends on sample quality, the parameters in the $\tilde{D}_{\text {dislocation }}$ model in Equation 12 can be different for different sets of samples. More systematic studies on the dislocation impact are necessary to establish a more generic model for $\tilde{D}_{\text {dislocation }}$.

\section{Conclusions}

In summary, this work focused on Si-Ge interdiffusion in epitaxial $\mathrm{SiGe}$ heterostructures with partial strain relaxation. As SiGe with strain relaxation is common for $\mathrm{SiGe}$ devices such as devices with $\mathrm{SiGe}$ heterostructures under biaxial strain, this work is of great relevance to the semiconductor industry. Based on diffusion theories, a model for point defect mediated Si-Ge interdiffusion $\tilde{D}_{\text {lattice }}(R)$ was established to include the impact from the degree of strain relaxation
$R$. Experimentally, structure $\mathrm{S} 4583$ with a metastable compressive $\mathrm{Si}_{1-y} \mathrm{Ge}_{y}$ layer was grown and annealed. Characterized by HRXRD, the compressive layer was shown to relax during the anneals, and $R$ value reached about $45 \%$ after the anneals. Furthermore, it was found that $R$ values reached a "plateau" quickly during the ramp-up stage of the anneals at $800^{\circ} \mathrm{C}$ and $840^{\circ} \mathrm{C}$. For these anneals, the strain relaxation happened before $\mathrm{Si}-\mathrm{Ge}$ interdiffusion. With the approximation that $R$ value is constant after the ramp-up stage of the anneals at $800^{\circ} \mathrm{C}$ and $840^{\circ} \mathrm{C}$, the $\tilde{D}_{\text {lattice }}(R)$ model gave close predictions of the Ge peak drop in the compressive $\mathrm{Si}_{1-y} \mathrm{Ge}_{y}$ layer. In addition, the impact of the dislocation mediated interdiffusion $\tilde{D}_{\text {dislocation }}$ was quantified, which is significant for interdiffusivity at lower Ge fractions. A two-term model that combines $\tilde{D}_{\text {lattice }}$ and $\tilde{D}_{\text {dislocation }}$ was shown to be very successful in explaining the data of Si-Ge interdiffusion with strain relaxation.

\section{Acknowledgments}

This work was funded by Natural Science and Engineering Research Council of Canada (NSERC), Crosslight Software Inc. and the University of British Columbia. We'd like to thank Prof. Judy L. Hoyt and Gary Riggott in the Department of Electrical and Computer Engineering at Massachusetts Institute of Technology for providing the epitaxial SiGe wafer in this work.

\section{References}

1. L. Gomez, C. N. Chleirigh, P. Hashemi, and J. L. Hoyt, IEEE Electron Device Letters 31(8), 782 (2010).

2. L. Gomez, P. Hashemi, and J. L. Hoyt, Electron Devices, IEEE Transactions on 56(11), 2644 (2009).

3. K. K. Bhuwalka, M. Born, M. Schindler, M. Schmidt, T. Sulima, and I. Eisele, Japanese Journal of Applied Physics Part 1-Regular Papers Brief Communications \& Review Papers 45(4B), 3106 (2006)

4. P. See and D. J. Paul, IEEE Electron Device Letters 22(12), 582 (2001).

5. Y. H. Kuo, Y. K. Lee, Y. S. Ge, S. Ren, J. E. Roth, T. I. Kamins, D. A. B. Miller, and J. S. Harris, Nature 437(7063), 1334 (2005).

6. S. J. Koester, J. D. Schaub, G. Dehlinger, and J. O. Chu, Selected Topics in Quantum Electronics, IEEE Journal of 12(6), 1489 (2006).

7. O. O. Olubuyide, Massachusetts Institute of Technology, 2007

8. J. Liu, X. Sun, R. Camacho-Aguilera, L. C. Kimerling, and J. Michel, Opt. Lett. 35(5), 679 (2010).

9. R. E. Camacho-Aguilera, Y. Cai, N. Patel, J. T. Bessette, M. Romagnoli, L. C. Kimerling, and J. Michel, Opt. Express 20(10), 11316 (2012).

10. G. M. Xia, J. L. Hoyt, and M. Canonico, J. Appl. Phys. 101(4), 044901 (2007).

11. G. R. Xia, H. M. Nayfeh, M. L. Lee, E. A. Fitzgerald, D. A. Antoniadis, D. H. Anjum, J. Li, R. Hull, N. Klymko, and J. L. Hoyt, IEEE Trans. Electron Devices 51(12), 2136 (2004).

12. J. W. Jung, S. F. Yu, O. O. Olubuyide, J. L. Hoyt, D. A. Antoniadis, M. L. Lee, and E. A. Fitzgerald, Appl. Phys. Lett. 84(17), 3319 (2004).

13. G. Dehlinger, S. J. Koester, J. D. Schaub, J. O. Chu, Q. C. Ouyang, and A. Grill, IEEE Photonics Technology Letters 16(11), 2547 (2004).

14. Jifeng Liu's personal communication (2010)

15. Y. Dong, Y. Lin, S. Li, S. McCoy, and G. Xia, J. Appl. Phys. 111(4), 044909 (2012).

16. Y. Dong, W. Chern, P. M. Mooney, J. L. Hoyt, and G. Xia, Semicond. Sci. Technol. 29(1), 015012 (2014).

17. J. H. Van Der Merwe, J. Appl. Phys. 34(1), 123 (1963).

18. J. W. Matthews and A. E. Blakeslee, J. Cryst. Growth 27, 118 (1974).

19. J. M. Hartmann, A. Abbadie, and S. Favier, J. Appl. Phys. 110(8), 083529 (2011).

20. Z. Da, T. White, and B.-y. Nguyen, Electron Devices, IEEE Transactions on 53(12), 3020 (2006).

21. C. P. Wong, J. Liu, J. Kasim, A. See, and Z. Shen, Jpn. J. Appl. Phys. 50, 040208 (2011).

22. P. M. Mooney, J. L. Jordan-Sweet, G. B. Stephenson, F. K. LeGoues, and J. O. Chu, presented at the Advances in X-Ray Analysis, 38: 43rd Annual Denver X-Ray Conference on application of X-Ray analysis, 1995 (unpublished).

23. P. M. Mooney, F. K. LeGoues, J. Tersoff, and J. O. Chu, J. Appl. Phys. 75(8), 3968 (1994).

24. PANalytical Epitaxy Quick Start Guide, Seventh Edition (January, 2007).

25. G. G. Fischer and P. Zaumseil, J. Phys. D: Appl. Phys. 28(4A), A109 (1995).

26. G. G. Fischer and P. Zaumseil, physica status solidi (a) 164(2), 767 (1997).

27. V. T. Gillard, W. D. Nix, and L. B. Freund, J. Appl. Phys. 76(11), 7280 (1994).

28. D. J. Dunstan, Journal of Materials Science: Materials in Electronics 8(6), 337 (1997).

29. S. de Gironcoli, P. Giannozzi, and S. Baroni, Phys. Rev. Lett. 66(16), 2116 (1991).

30. B. Dünweg and D. P. Landau, Physical Review B 48(19), 14182 (1993).

31. J. W. Cahn, Acta Metall. 9(9), 795 (1961).

32. J. W. Cahn and J. E. Hilliard, The Journal of Chemical Physics 28(2), 258 (1958). 
33. M. J. Aziz, Y. Zhao, H.-J. Gossmann, S. Mitha, S. P. Smith, and D. Schiferl, Physical Review B 73(5), 054101 (2006).

34. A. L. Greer and F. Spaepen, Synthetic Modulated Structures. (Academic Press, Orlando, 1985).

35. Synopsys, Inc., 2007 Dec.

36. Z. Erdélyi, I. A. Szabó, and D. L. Beke, Phys. Rev. Lett. 89(16), 165901 (2002)

37. D. B. Aubertine, M. A. Mander, N. Ozguven, A. F. Marshall, P. C. McIntyre, J. O. Chu, and P. M. Mooney, J. Appl. Phys. 92(9), 5027 (2002).
38. P. M. Mooney and J. L. Jordan-Sweet, JCPDS-International Centre for Diffraction Data (1999).

39. J. H. Basson, H. Booyens, and C. A. B. Ball, J. Appl. Phys. 50(6), 4003 (1979).

40. X. W. Liu, A. A. Hopgood, B. F. Usher, H. Wang, and N. S. J. Braithwaite, Semicond Sci. Technol. 14(12), 1154 (1999).

41. J. S. Christensen, A. Y. Kuznetsov, A. E. Gunnaes, B. G. Svensson, and H. H. Radamson, Materials Science in Semiconductor Processing 9(4-5), 650 (2006).

42. M. Gavelle, E. M. Bazizi, E. Scheid, P. F. Fazzini, F. Cristiano, C. Armand, W. Lerch, S. Paul, Y. Campidelli, and A. Halimaoui, J. Appl. Phys. 104(11), 113524 (2008). 